\title{
AN OPERATING AND OBSERVATION URETHROSCOPE.
}

THIs instrument was designed as a simplification of the existing aero-urethroscopes, and is one that allows greater facilities for intra-urethral operations under air distension than was previously possible.

The urethroscope follows the usual type of instruments with "external illumination," the light from the electric bulb being focused, by a condenser, on a mirror set at the angle of 45 degrees. By this it is reflected down the urethral tube. The observer looks through a hole in the centre of the mirror. In many details, hnwever, this urethroscope departs from the design of other instruments. It consists of a T-shaped tube. One horizontal limb of the $T$ is conical, and ends in a nozzle that fits the standard urethral tubes now in use. The other horizontal limb is very short and is slotted to receive the "eye-piece." The latter is a strong metal ring that fits accurately into the horizontal tube, and is held in place by two small binding screws. Posteriorly it carries the window through which the observer looks. This window occupies the upper twothirds of the circle, while the lower third is pierced by an aperture through which the instruments are inserted for the purpose of operation. When the urethroscope is used for observation this aperture is closed by a small metal cap, which engages by means of a bayonet catch. In front of this window the eye-piece carries a strong metal frame to which the mirror is attached. This is so adjusted that when the eye-piece is in place the mirror reflects the light exactly down the urethral tube. This adjustment is effected before the instrument leaves the factory, and as the eye-piece is so strongly made no ordinary rough usage can alter the position of the mirror. The vertical limb of the $\mathrm{T}$ contains the lamp-holder, and at the junction of the two limbs the condensing lens is fixed. The lamp-holder slides within the vertical tube for the purpose of focusing ; the movement is controlled by means of a stud that runs in a spiral slot. This is the only adjustment that need be made by the observer, and that only when a new lamp is fitted. The lamp, condenser, and mirror are indicated in the illustration by stippling. (See Fig. 1.)

The operation attachment is also extremely simple, and practically any type of instrument can be used with this urethroscope. I have designed a probe, knife, curette, electrolysis needle, and an electric cautery to fit it. All these instruments have a double bend on the shaft to allow

FIG. 3.

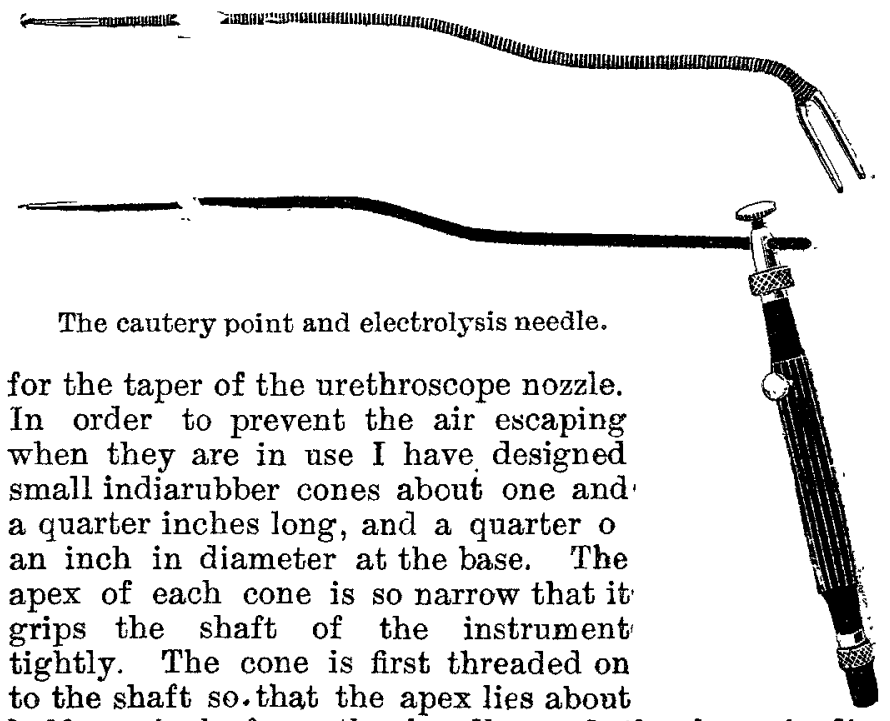

half an inch from the handle, and the base is directed towards it. The base of the cone is next seized and pulled forward over its apex. This has the effect of turning the wider part of the cone inside out, and into it the apes becomes invaginated, as in Fig. 2. The base of the cone is

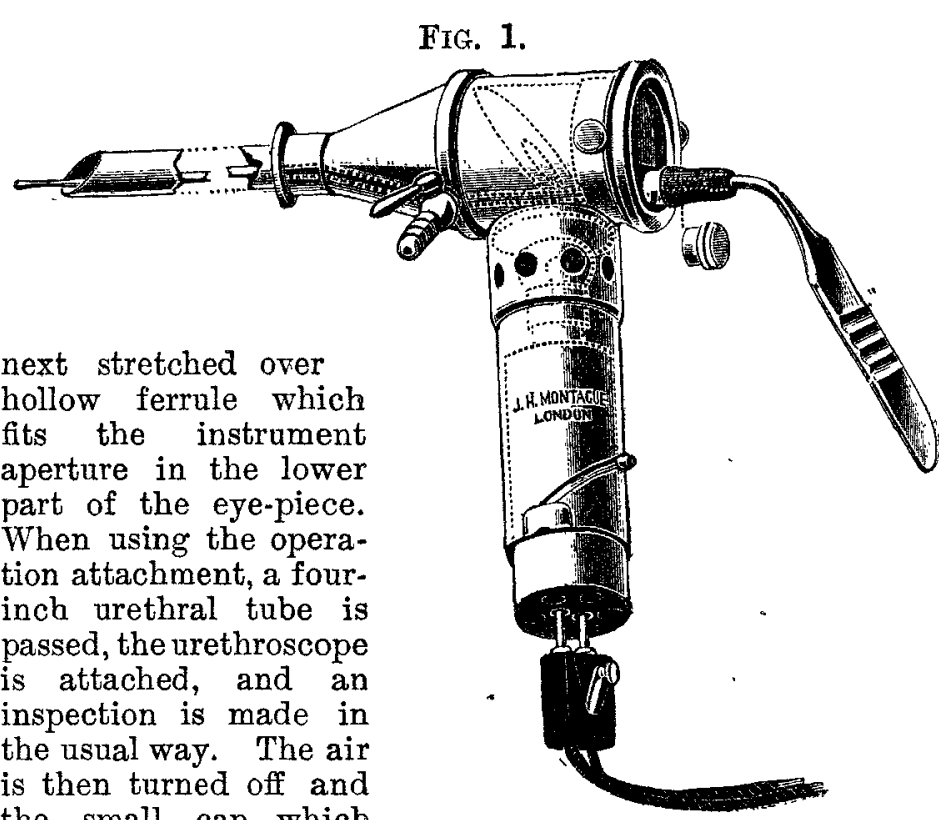

is then turned off and the small cap which closes the "instrument aperture" is removed. The required instrument is now passed through the aperture, and the ferrule on the cone is engaged in the bayonet catch on the
FIG. 2

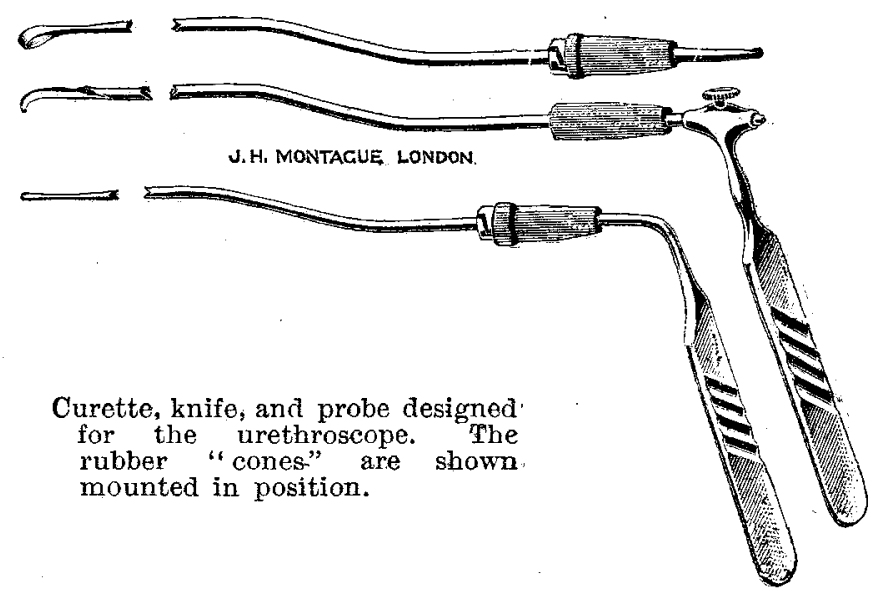
eye-piece. Finaily the air is again turned on. The cones are threaded over the points of the instruments, except in the case of the knife and curette, where it is obviously impossible to do so. Both these instruments are provided with detachable handles for this purpose. Once a cone is adjusted on the shaft of an instrument, it need not be touched until the rubber is perished, and it is necessary to replace it. By means of this contrivance one gets a forward and backward range of movement of at least one inch free lateral movement, and an air-tight joint. As long as the apex of the cone is.invaginated, the greater the air pressure, the more secure the joint.

Amongst the adwantages of this instrument are the following. 1. Simplicity;: only one adjustment need be made, and that only when a new lamp is fitted. 2. Durability; the mirror, lens, and all vulnerable parts are enclosed and protected from damage. 3. Good illumination is obtained; as the urethroscope is so compact the object is always near the observer's eye. At the same time there is a total absence of troublesome reflections. 4. The ease with which the operating instruments can be inserted or changed. 5. Freedom of movement and delicacy of touch are retained when operating under air distension.

The urethroscope and instruments have been made for me by Mr. J. H. Montague, New Bond-street, London, W.

Harley-street, $W$. J. SWIFT JoLY, F.R.C.S..Eng.

Pogt-Graduate Course on the Feeding and CARE OF INFANTS. - In view of applications for permission to attend separate lectures of this course, the National Association for the Prevention of Infant Mortality has decided to issue tickets at $1 s$. each for any single lectures that students may desire to attend. Prospectuses and tickets may be obtained from the secretary, 4 , Tavistock-square, London, W.C. 